\title{
ACTIVITÉS SPORTIVES ET « DÉCONTRÔLE » DES ÉMOTIONS
}

Esquisse d'une analyse des usages de produits psychoactifs dans le sport et hors le sport

\section{Sylvain Aquatias}

Médecine \& Hygiène | «Déviance et Société »

2003/3 Vol. 27 | pages 313 à 330

ISSN 0378-7931

Article disponible en ligne à l'adresse :

https://www.cairn.info/revue-deviance-et-societe-2003-3-page-313.htm

Distribution électronique Cairn.info pour Médecine \& Hygiène.

(C) Médecine \& Hygiène. Tous droits réservés pour tous pays.

La reproduction ou représentation de cet article, notamment par photocopie, n'est autorisée que dans les limites des conditions générales d'utilisation du site ou, le cas échéant, des conditions générales de la licence souscrite par votre établissement. Toute autre reproduction ou représentation, en tout ou partie, sous quelque forme et de quelque manière que ce soit, est interdite sauf accord préalable et écrit de l'éditeur, en dehors des cas prévus par la législation en vigueur en France. Il est précisé que son stockage dans une base de données est également interdit. 


\section{ACTIVITÉS SPORTIVES ET «DÉCONTRÔLE» DES ÉMOTIONS Esquisse d'une analyse des usages de produits psychoactifs dans le sport et hors le sport}

Sylvain Aquatias*

Mots-ClÉS: PRODUITS PSYCHOACTIFS - ACTIVITÉS SPORTIVES - ÉMOTIONS - MAÎTRISE DE SOI

KeY-WORDS: DrugS - SPORTS - EMOTIONS - SELF CONTROL

Le présent texte se base sur une recherche qualitative commanditée par le ministère de la Jeunesse et des Sports (Aquatias (dir.), 1999), qui avait pour objectif d'examiner d'un point de vue qualitatif le rapport entre conduites à risques et pratiques sportives. Cette recherche gravite autour d'une question que l'on peut formuler ainsi: dans quelles conditions les activités sportives sont-elles susceptibles d'être des facteurs de protection ou de vulnérabilisation en matière de consommation de produits psychoactifs et/ou dopants et, plus globalement, de conduites à risques des jeunes?

Pour mieux comprendre quelle articulation existe entre pratiques sportives et conduites à risques, nous avons volontairement cherché des situations où les sportifs rencontrent différents problèmes. Il ne s'agit donc en aucun cas d'une analyse quantitative qui évaluerait la prévalence des risques en milieu sportif. Cinq pôles de recherche ont été choisis:

1. Des jeunes de milieu populaire participant à des activités sportives de vacances (11 entretiens plus 2 entretiens collectifs et des observations participantes).

2. Des jeunes participant à des activités d'insertion par le sport (15 entretiens).

3. Des consommateurs de produits psychoactifs pratiquant en clubs (9 entretiens).

4. Des personnes étant passées par les filières sport études (10 entretiens).

5. Des personnes ayant eu un parcours dans le sport de performance et dans la toxicomanie (11 entretiens).

Des entretiens complémentaires ont été faits avec des dirigeants de club et des entraîneurs chaque fois que cela était nécessaire. Le recrutement des personnes interrogées a été effectué soit par l'intermédiaire d'institutions (pour les pôles 1, 2 et 5), soit par des réseaux informels, soit sur la méthode dite de boule de neige (3 et 4 ).

On notera que de multiples croisements se font entre les cinq pôles choisis: certains sportifs en club sont passés par les sport études, certains jeunes rencontrés dans les activités sportives de vacances sont aussi en club, etc. Les sports représentés ainsi que les niveaux de jeu sont très diversifiés et ne présentent pas de récurrence particulière.

* IUFM Limoges. 
À partir de ces entretiens, nous avons reconstitué des trajectoires de vie les plus précises possibles, en examinant la conjonction des différentes périodes et événements à travers les domaines de la vie familiale, de la vie scolaire et professionnelle, de l'activité sportive et enfin du parcours de santé. Les usages de produits psychoactifs ou/et dopants apparaissent clairement dans ces reconstitutions biographiques en conjonction avec des moments du calendrier sportif ou des périodes de la vie des personnes interrogées. C'est ce qui m'a incité à étudier ici le lien entre les usages de produits psychoactifs et les différentes activités sportives. D'ailleurs, parmi les diverses fonctions sociales de ces deux pratiques, une leur est commune: elles contribuent à la libération des émotions.

\section{Libérer les émotions: activités mimétiques et désinhibition chimique}

Notre société est une société de maîtrise de soi (Elias, 1973, 1975). La retenue et la mesure sont devenues des conditions permettant la vie en société. D'ailleurs, la plupart des débordements passionnels font l'objet d'un traitement social, qu'il soit individuel ou collectif. On réprime les émeutes naissant de l'émotion consécutive à la mort d'un jeune dans les quartiers «sensibles » ou les excès de supporters trop enthousiastes après un match, on donne à l'enfant trop turbulent un calmant ou la consigne d'aller se défouler un peu en faisant du sport.

Il est capital qu'existent des moyens de libération des émotions. Celles-ci, trop longtemps retenues, peuvent s'exprimer brutalement dans des débordements impromptus qui remettent en cause l'ordre social. Il faut donc que se constituent des activités où le contrôle de soi diminue et où l'expression d'émotions est possible, sans que celles-ci ne débordent le cadre qui leur est donné pour atteindre d'autres activités de la société.

Cependant, l'extension de la maîtrise de soi et l'intensité avec laquelle elle s'applique ont provoqué une intériorisation profonde des mécanismes de contrôle des impulsions. Le relâchement des émotions n'est alors plus évident. Il est donc tout aussi nécessaire qu'existe dans ces espaces un support ou un stimulus fort qui fasse naître des émotions socialement acceptables dans ce cadre.

Autrement dit, les moyens de se délivrer de la tension permanente du contrôle de soi ne peuvent prendre corps que dans des activités où les émotions sont à la fois stimulées, acceptées et canalisées.

Les activités de loisir ont cette fonction (Elias, Dunning, 1994). Parmi ces activités, on trouvera aussi bien des activités sportives qu'artistiques, toujours liées aux loisirs et donc dénuées d'enjeux forts. Norbert Elias les appelle des «activités mimétiques», parce qu'elles évoquent suffisamment des situations de la vie courante pour que des émotions proches de celles retenues dans la quotidienneté surgissent. L'identification du spectateur au personnage d'un film ou d'une pièce de théâtre, l'effort du sportif amateur ou la concentration de l'auditeur de musique entrent dans cette classe d'activités.

Pourtant les activités mimétiques ne sont pas les seuls moyens de favoriser l'émergence des émotions habituellement retenues. Les produits psychoactifs comme l'alcool, le cannabis, l'ecstasy, etc., en sollicitant les centres nerveux, provoquent une désinhibition toute chimique. Si on les consomme dans les réunions entre amis et dans beaucoup de festivités, c'est bien qu'ils facilitent le contact et l'émotivité en neutralisant le contrôle de soi qui nous fait garder des distances et des réserves. La conjonction entre réunions amicales et consommation de produits psychoactifs est tellement fréquente que de nombreux chercheurs parlent d'usages «récréatifs» ou «festifs» pour les désigner. D'ailleurs, la 
recherche de communication se retrouve parmi les motivations principales de consommation de produits psychoactifs dans le milieu Techno (Aquatias, 1998-1999). L'alcool ou le cannabis, s'ils ne se limitent pas à ces fins, sont assez fréquemment utilisés pour augmenter la sensibilité émotionnelle à un événement, qu'il s'agisse d'un mariage, d'un concert (Aquatias (dir.), 2002), voire d'un repas de famille ou d'une soirée entre amis.

Il n'est bien sûr pas question de limiter ici les utilisations de ces produits à la libération émotionnelle, mais simplement de souligner que leur consommation offre une alternative aux activités mimétiques, voire se joint à elle dans certaines situations. Activités mimétiques et consommation de produits psychoactifs sont deux moyens possibles de se débarrasser du contrôle de soi qui nous empêche d'exprimer nos émotions. Les unes fournissent un support à l'émotion en reproduisant des caractéristiques des activités où les pulsions sont contrôlées, les autres sensibilisent et désinhibent, rendant l'expression des émotions possibles, y compris hors d'un cadre spécifique. Pourtant, de la même manière que l'on ne peut limiter le rôle des produits psychoactifs à la désinhibition, l'on ne peut réduire la fonction des activités sportives à la libération des émotions.

\section{Trois fonctions sociales des activités sportives}

Les activités sportives remplissent de nombreuses fonctions sociales qu'il est impossible d'étudier en intégralité ici. Trois de ces fonctions sont particulièrement importantes en ce qui concerne les modes de rétention et d'expression des émotions. Le sport, en tant qu'activité mimétique, permet d'estomper le contrôle de soi et favorise l'expression d'émotions. C'est la première fonction. La seconde fonction concerne la possible professionnalisation des pratiquants. La troisième remplit une fonction éducative par l'apprentissage de la rétention des émotions et de la discipline, notamment auprès des jeunes.

La première fonction, la libération du contrôle de soi, peut s'effectuer aussi bien par le spectacle sportif que par l'activité elle-même. Il faut, pour que l'activité sportive participe réellement au relâchement émotionnel, qu'elle n'ait pas d'incidence forte sur la vie «hors sport», et se bâtisse en rupture avec la routine quotidienne. S'il est nécessaire que les joueurs croient au jeu pour que surgissent les stimuli permettant le «décontrôle» émotionnel, il est tout aussi nécessaire que ceux-ci sachent que les résultats du jeu ne vont pas changer leur vie.

De même que pour la pratique sportive, le spectacle sportif crée une rupture avec la vie quotidienne, fournit un stimulus sur la base d'une compétition entre individus ou entre équipes et les enjeux ne peuvent changer réellement la vie des spectateurs. Mais aussi, pour que continuent à se produire des stimuli suffisants, il faut que les performances s'élèvent audessus du commun et que le suspense soit toujours plus important. Le spectacle sportif est tenu de surenchérir sans cesse sur lui-même. À ce point se surajoute la nécessité pour les villes et les pays de défendre leur honneur sur la scène sportive (Elias, Dunning, 1994).

Reste qu'il faut bien produire ces sportifs qui vont enchanter les foules. C'est là la deuxième fonction. Une classe de sportifs volontaires et motivés, entraînés depuis la plus petite enfance et ayant parfaitement intériorisé les valeurs du jeu sportif, doit être formée. Il n'est plus là question de relâchement des émotions, mais au contraire d'effort incessant, de plaisir différé, de discipline et d'autocontrôle. Mais cette classe émerge directement de la masse des pratiquants. Même s'il existe des différences selon les sports, les fédérations, 
les clubs et les entraîneurs, les schémas d'intériorisation des valeurs sportives liées à l'abnégation et à la discipline des corps semblent se diffuser de manière constante. Le modèle même de la réussite sportive diffusée par les médias s'impose aux jeunes à qui leurs premières performances permettent quelques espoirs. De fait, sans même aller jusqu'à une réelle professionnalisation, les activités sportives sont tellement investies par certaines personnes qu'elles s'attachent ainsi un statut social parfois important, qu'elles s'imposent un rythme de vie correspondant à cette pratique (ce qui s'oppose à l'idée de rupture de la routine quotidienne), parfois même obtiennent ainsi des revenus complémentaires. Entre le sport comme loisir et le sport comme activité quasiment principale, la différence peut alors devenir bien mince.

La troisième fonction sociale du sport liée aux émotions concerne les enfants et les adolescents: les activités sportives sont supposées être un moyen d'éducation des jeunes. Si l'activité sportive permet le relâchement des émotions pour des adultes dont le comportement dans la vie quotidienne se doit d'être mesuré, elle est aussi, pense-t-on, un excellent moyen pour les jeunes de faire l'apprentissage d'un contrôle de soi qu'ils maîtrisent encore mal. Les pratiques mimétiques créent un cadre où les émotions sont à la fois stimulées et canalisées: si, au niveau des adultes, c'est la stimulation des émotions qui joue, au niveau des jeunes, c'est la canalisation qui est sensée intervenir. L'apprentissage des limites du relâchement des émotions serait transféré de l'activité sportive vers la vie quotidienne. Moyen de socialisation, le sport permet aux enfants et aux adolescents de développer un potentiel d'autodiscipline face au déchaînement soudain de leurs pulsions et affects, et ce dans le cadre d'une communauté humaine, selon des normes précises de régulation des conduites et des sentiments (Elias, 1996, 29).

Pour récapituler, le sport permet d'éduquer les jeunes générations, de créer des espaces de libération des émotions pour les adultes, d'atteindre à la reconnaissance sociale en s'inscrivant dans un parcours de performance. En fait, selon les types d'activité sportive et les différents investissements des personnes dans ces activités, plusieurs de ces fonctions peuvent se retrouver à des degrés différents dans une même pratique. Néanmoins, deux de ces fonctions sociales, l'aspect éducatif et l'aspect libératoire, devraient procéder par mimétisme avec la vie quotidienne et limiter ainsi les éclats émotionnels.

L'apprentissage de la rétention des émotions par le sport semble pourtant ne fonctionner que de manière très aléatoire, que ce soit dans le spectacle sportif où les débordements des supporters sont fréquents, ou dans la pratique, pour les compétiteurs de haut niveau qui se laissent parfois aller à la violence ou pour les jeunes qui pratiquent le sport à petit niveau. Les débordements varient bien sûr selon les sports et certains semblent plus propres à déchaîner les émotions sans qu'elles soient canalisables. Mais, au delà de la scène de la compétition, les effets pacificateurs de l'activité sportive ne semblent pas si nets. Les sportifs, jeunes ou moins jeunes, pratiquant à haut niveau ou non, ne semblent pas avoir des comportements si tranquilles dans les activités «hors sport». De même, puisque les activités sportives sont supposées favoriser l'expression des émotions, elles pourraient, ne serait-ce qu'en partie, limiter les usages de produits psychoactifs liés à cette même fonction.

En considérant les différents usages qui sont faits des produits psychoactifs, ne peut-on pas trouver des clés qui puissent permettre de comprendre pourquoi les effets de libération ou de rétention des émotions du jeu sportif fonctionnent peu? Il nous faut alors observer comment s'articulent les différents usages de produits psychoactifs et les différents usages du sport. 


\section{Les différents usages de produits psychoactifs dans les activités sportives}

Chaque fois que des groupes de jeunes se forment, une forme de convivialité se développe, qui peut aboutir à des échanges et des expérimentations dans le groupe. Que certains jeunes aient pu fumer leurs premières cigarettes, leurs premiers joints ou connaître leurs premières ivresses dans des sociabilités liées à l'activité sportive n'a donc rien d'étonnant. S'il est cependant une expérimentation qui est plus propre aux milieux sportifs, c'est celle qui lie «défonce» et compétition. La plupart du temps, les jeunes expérimentateurs choisissent une compétition sans grand enjeu. Mais, parfois aussi, c'est bien le fait de doubler la consommation avec l'usage sportif qui est recherché, en ce sens qu'il permet de s'attribuer une identité positive plus forte: on a tenu le coup, on a réussi à jouer, même «défoncé». On trouve dans ce type d'expérimentation des prises de cannabis, d'alcool, d'amphétamines et même un cas de produit dopant.

Donc une fois, aux championnats, avec un copain, pour rigoler, on a pris un truc... On a cherché dans la liste des produits interdits (...) Moi, c'était pour voir ce que ça pouvait donner... (...) C'étaient des petites pilules...(...) On me l'avait présenté comme un truc utilisé dans le cyclisme... En fait, j'avais un copain qui était médecin, c'est lui qui me l'avait filé... On a fait un essai à l'entraînement. On se sentait bien (rires)... On était léger, on n'avait pas de perte de rythme...

L'expérience se terminera mal, les deux expérimentateurs se retrouvant tétanisés avant la fin de la compétition. Mais l'expérimentation de certains produits dopants se fait parfois aussi de manière plus institutionnelle: une sportive se souvient ainsi d'avoir été malade à douze ans après avoir pris du Guronsan ${ }^{1}$ que son entraîneur lui avait donné et une directrice de club avoue qu'elle fait un dopage contrôlé de ses juniors, pour essayer de répondre à leurs propres exigences de compétition.

En dehors des expérimentations, qu'elles concernent des produits répondant à la recherche de performance ou des produits produisant des effets euphorisants, on trouve de nombreux types d'usages.

Certains des pratiquants rencontrés qui ont des habitudes de consommation régulières mettent en œuvre un certain nombre de régulations. Un fumeur quotidien de cannabis sacrifie ainsi ses habitudes quand il va s'entraîner dans son club:

Disons que généralement, j'évite quoi... De temps en temps, (...) je fume l'après-midi, et je ne pense pas que j'ai sport le soir... Je suis avec des potes, paf, voilà, ça m'arrive de fumer un joint quoi... Ou bien je sors de chez un pote et je vais direct au club, voilà quoi...

Un autre sportif, d'un plus haut niveau, raconte qu'ayant changé de club et donc de ville, il se retrouve un peu isolé et prend un logement avec deux autres sportifs, venant eux aussi d'arriver. Leur consommation de cannabis et de tabac devient «presque» quotidienne. Pourtant, lors des stages et des compétitions, il est attentif à ne pas faire d'excès :

Médicament tonique, prescrit en traitement de l'asthénie fonctionnelle, pouvant entraîner une excitation psychomotrice, de l'insomnie et des palpitations, contenant du glucuronamide, de l'acide ascorbique et de la caféine. 
Quand j'étais en équipe de France, en général ça diminuait beaucoup... Entre trois et cinq par jour, les cigarettes. Et les pétards, pratiquement pas, quoi...

Un sportif de haut niveau résume bien ces régulations et leur motivation: Le cannabis, ce n'était pas avant l'entraînement, non c'était peut-être le soir, allez, hop, j'avais fini ma journée, je me mettais devant un film, on était avec trois, quatre copains, on se mettait une cassette vidéo, et puis voilà, tranquille. Ce n'était pas pour dire "demain je vais être reposé, je vais faire une performance». Non, c'était comme ça pour décompresser tout simplement...

En plus là, c'est une période, ça fait cinq jours, on fait la fête tous les soirs, parce que ça y est, on n'est pas qualifié... La saison est terminée, on est en mai, explique un autre joueur.

Ces situations ne sont certes pas spécifiques aux sportifs (pas plus qu'elles ne se trouvent chez tous les sportifs) et l'on retrouve partout en société des usages similaires.

Encore faut-il ici relativiser. Car certains consomment aussi tabac, alcool et cannabis pendant les périodes de compétition.

- À quel moment tu as été sélectionné pour ces stages?

- Les premiers stages, je devais avoir seize ans... J'en avais un par an... National... Et j'ai été sélectionné toutes les années entre la seconde et le bac...

- Vous étiez nombreux à fumer et à boire comme ça?

- Non, peu... On était cinq ou six... Mais bon, c'était déjà pas mal, je trouve... Parce que, bon, pour moi, ce n'était pas un truc habituel non plus, mais pour eux, c'était un moyen pour se mettre en préparation...

Et on retrouve ailleurs des joueurs qui, à l'occasion, fument un joint avant un entraînement ou un match.

- Et dans ton équipe, il y avait des gars qui fumaient?

- Ouais, ouais... Oui, ben... Régulièrement, certains jours, avant d'aller taper dans le ballon, on fumait quoi... Avant les matchs, même...

Les connivences qui peuvent relier les consommateurs de haschich peuvent d'ailleurs faciliter les rencontres et les relations lors des stages.

- C'est-à-dire qu'on avait des stages nationaux où ils en prenaient trente susceptibles de faire l'équipe de France... Et donc là on s'est retrouvé avec d'autres gens de la France entière, et c'est là que j'ai vu qu'y en avait qui fumaient, ou qui...

- Du tabac ou...

- Ah non, shit...

- Tu t'en es rendu compte comment?

- Ah ben parce que ça se voit quoi... Et puis en plus, on discutait, et comme moi, je leur avais dit que je fumais aussi, donc il arrivait que je fume un peu avec eux aussi. On se barrait en ville pour aller dans les bars le soir... Tout ça... Il s'était créé une petite amitié comme ça, mais juste par rapport à ça... Parce que dès qu'on était sur la piste (...), je retrouvais cette agressivité de vaincre. 
Les encadrants ne semblent guère prêter attention à ces consommations.

Par rapport aux entraîneurs, c'était caché... Euh... Caché, c'est un bien grand mot... Disons que ça devait se savoir, les entraîneurs fermaient plus ou moins les yeux, sans doute... (...) Et l'entraîneur de X, qui était en équipe de France, il le savait, mais on ne fumait pas devant eux, on faisait attention, on était discret quoi... Les joueurs le savaient, mais on était discret, ceux qui voulaient fumer fumaient, et les autres... Et quand on fumait, on restait avec des gens qui fumaient... C'était jamais en groupe, c'était toujours à deux ou trois...

Bien sûr, il est parfois difficile pour ceux des sportifs rencontrés qui sont eux-mêmes consommateurs de cannabis de faire la morale aux jeunes qu'ils entraînent. Parfois même, les jeunes connaissent les habitudes de consommations de leur entraîneur.

- Comment ça se passe le cannabis dans ton équipe?

- Y'a rien de caché, l'entraîneur, c'est un fumeur, donc...

- Quelles sensations apportent le cannabis lors du jeu? Peut-il favoriser la performance?

- Je pense que ça influait sur mon match... Au sens où je ne me prenais pas au sérieux, il y avait une pseudo-complicité avec le type avec qui j'avais fumé qui, lui, comprenait que si j'avais loupé tel truc... Ça n'atténuait pas énormément ma performance, mais ça agissait plus sur ma réaction. Au lieu de mettre un coup de poing par terre et de réagir à un truc que j'aurais loupé par exemple, c'était rire et tout de suite rechercher le regard complice de l'autre avec qui j'ai fumé, parce que lui peut l'interpréter, explique un footballeur de niveau régional.

Un escrimeur, jouant au niveau national, complète:

- À un moment, je fumais avant ou pendant toutes les compétitions. J'étais bien, sans stress, j'y allais comme ça quoi... Et puis, ça me détendait quoi.

S'il n'y a pas là de recherche de performance, il y a bien une quête de relaxation par rapport à la tension de l'épreuve compétitive. Le même sportif raconte avoir perdu un match «gagné d'avance» dans une importante compétition pour s'être laissé déstabiliser par la peur.

Pour les sportifs de bon niveau, cette recherche de détente se retrouve aussi dans des usages assez intensifs de produits, qu'il s'agisse alors d'alcool, de cannabis ou même d'héroïne, de cocaïne et d'ecstasy, lorsque s'arrête la tension liée à la compétition sportive.

On sort peu, mais quand on sort, c'est intense, disent plusieurs athlètes. Ces excès sont relativement limités dans les sports individuels et assez fréquents dans les sports collectifs.

- On avait plus de matchs, alors on sortait... Quand on était jeunes, c'était souvent les boîtes...

- Toujours après les victoires?

- (sourire) Toujours. Après les victoires ou les défaites...

explique un joueur de rugby. 
L'effort intense que font les sportifs pendant les temps d'entraînement trouvent là leur correspondance en termes de décompression. C'est ce qu'illustre bien l'extrait suivant d'entretien.

(...) Sans cette décompression, de toute façon, je ne pense pas que j'aurais pu aborder l'entraînement comme je l'abordais. Moi, je le voyais comme ça. Le fait d'avoir pris entre guillemets une bonne caisse, hop, j'arrivais, allez, bon, on attaque à nouveau, ça repart à zéro. Je le voyais comme ça et j'étais consommateur de fêtes. Consommateur de fêtes et j'en avais même besoin. Je vais le dire comme ça: «j'en avais besoin ». C'est dur de rester tout le temps dans le même système et c'est pour ça aussi que l'on explose. Parce que généralement avant les compétitions, un mois, deux mois avant les compétitions, là, vous ne faites plus de sorties quand c'est une échéance vraiment importante, là il n'y a plus de consommation de cannabis, il n'y a plus de consommation d'alcool, il n'y a plus tout ça.(...) Le jour où cette compétition-là, elle est finie, là, c'est une explosion, c'est-à-dire qu'il n'y a plus de limites, c'est-à-dire que ça explose. C'est les abus, il y a des abus dans tout, c'est clair. Des abus dans tout et je serais presque tenté de dire pour tout le monde, presque. (...) C'est facile de craquer donc, on se retient à ce qu'on peut.

Les prises de produits, on le voit, suivent en général les temporalités de la pratique sportive: fins de journée, fins de compétition, fins de saison, etc. Elles correspondent à une célébration autant qu'à une recherche de décompression. Pour fêter une victoire, on consommera beaucoup plus. (...) Comme on était sur le podium en double, on était quatre sur six à être sur le podium complètement défoncées! dit une sportive en riant (il s'agit de cannabis). Pour se détendre de la journée passée, on boira quelques verres ou l'on fumera un joint ou deux, pour arroser une victoire importante ou la fin de la saison, on se laissera aller à des excès notables. Ces festivités ne sont pas toujours directement liées au milieu sportif, mais elles prennent corps dans la conjonction entre la fin de l'activité sportive et le relâchement du contrôle des émotions.

Les usages correspondent à des logiques de décompression et les pratiquants en parlent comme d'un besoin. Ils sont d'autant plus importants que le niveau de compétition s'élève. $\mathrm{Au}$ fur et à mesure que les attentes des institutions et des sportifs vis-à-vis d'eux-mêmes augmentent et que la pression crôt, les espaces de décompression semblent devenir plus intenses et/ou plus fréquents. Parce qu'elles correspondent aux temporalités du calendrier sportif, elles peuvent, en fin de saison, durer plusieurs jours et se répéter pendant toutes les «vacances».

Les usages de produits psychoactifs liés à la performance, eux, sont en fait davantage des usages de stimulants: caféine, amphétamines, cocaïne, etc.

Une adepte des jeux de raquette, qualifiée au niveau national, l'explique bien:

(L'effet du cannabis), ça dépendait, ça n'influençait pas le jeu. Si on était bien dans sa tête avant, ça allait bien. Par contre, si j'étais mal, parfois ça m'apportait un petit bien-être quand même, mais je ne peux pas dire que c'est le produit à prendre dans ce sport! Par contre, ça m'est arrivé une fois de jouer sous amphétamines, là oui, c'est plus un produit pour mon sport, parce que, comme ça speede, on a l'impression d'avoir beaucoup plus de temps pour se déplacer sur la balle. Elle ne reproduira pas cette expérience: Ça se voyait trop, dit-elle.

Un joueur de rugby en sélection régionale raconte aussi comment il a usé et abusé de caféine et de vitamines. 
- À une époque, je carburais au Guronsan et tout ça, aux vitamines, mais bon, j’ai arrêté, parce que ça me bousillait plus qu'autre chose, j'étais tout le temps à fond...

- Tu carburais... C'est-à-dire?

- Trois, quatre Guronsan par jour, avec deux, trois vitamines C de temps en temps. Je me suis rendu compte que je dormais très peu...

- Ça a duré longtemps?

- Ouais, c'était l'année dernière en début de saison, parce que je ne m'étais pas préparé du tout les deux mois d'été.

Et un footballeur raconte un autre type de consommation:

On jouait un match, c'était la première fois qu'on se qualifiait pour éventuellement envisager de monter en première division. Ce jour-là, je n'étais pas bien et on m'a donné (un entraîneur) un médicament qui s'appelait du Captagon ${ }^{2}$, qui est un médicament qui te fait monter à 5000 ... (...) Enfin, je ne sais pas ce que c'est exactement, mais c'est un excitant... Enfin, quand je l'ai pris, au bout d'une demi-heure, trois quarts d'heure, dans les vestiaires, quand on se changeait, j'ai commencé à avoir chaud de partout, je me suis mis à suer... Et puis sur le terrain, je partais d'un bout à l'autre du terrain, pas de sensation de fatigue ni quoi que ce soit... (...) Comme un malade quoi... J'ai été blessé durant ce match, il y a un type qui m'a marché dessus avec ses crampons, c'était sur la cuisse, j'avais une estafilade, mais très impressionnante, qui saignait et je n'ai rien senti... Donc, j'étais vraiment dans un état second... (...) Je suis sorti du terrain, j'avais toujours envie de courir. Le soir, on a fait la fête comme des fous... Toujours sans rien sentir. L'alcool là-dessus, ça a été un cocktail assez explosif... Bilan: je suis rentré chez moi, j'ai mis un jour et demi pour m'en remettre, quoi...

Nous sommes là face à des usages qui sont de l'ordre, clairement, du dopage, même si leurs rapports à la performance ne sont pas les mêmes. Dans le cas de l'équipe de football, le doute n'est pas permis: face à une situation d'incertitude, il est nécessaire de «renforcer» les capacités sportives des joueurs. Pour le joueur de rugby, les choses sont tout autres: n'ayant pu s'entraîner pendant les vacances et craignant de ne pas être à la hauteur, il va recourir à des produits susceptibles de le «remettre en forme». Dans un cas, il s'agit d'augmenter les performances pour monter au classement, dans l'autre, il s'agit de ne pas voir baisser ses performances.

Enfin, il reste un autre usage des produits psychoactifs qui se lie parfois aux pratiques sportives: la toxicomanie. Nous avons rencontré onze sportifs par l'intermédiaire des structures de traitement pour toxicomanies. Seuls quatre d'entre eux ont consommé pendant leur carrière sportive, les autres à la suite de l'arrêt de celle-ci.

Le 8 septembre 88, j'ai arrêté le sport, j'ai arrêté les études en même temps, j'ai tout arrêté. Je me suis dit "si en moi, ça recherche l'échec, c'est plus la peine...». J'ai vraiment décompressé: les produits, j'en avais déjà pris avant, mais là, ça a vraiment été la défonce, vraiment la défonce. (...) Après j’ai plus rien fait. 88, encore une tentative de suicide, je me suis réveillée à l'hôpital psychiatrique, explique une sportive.

2 Psychotonique à action centrale de la classe des amphétamines, dont la commercialisation a été arrêtée en 1993. 
Tous, sauf un, ont été des sportifs de bon niveau, sinon de haut niveau et l'on sait que l'arrêt du sport est toujours difficile dans ce contexte.

Leurs premières consommations ont souvent lieu au cours de festivités, boîte de nuit ou soirées privées, sans que celles-ci soient nécessairement liées aux sociabilités sportives.

Mais plusieurs d'entre eux ont comparé les sensations sportives et celles procurées par les produits psychoactifs. D'abord, au niveau défoulement, c'est énorme. (...) Et puis l'idée d'avoir fait ce qu'il fallait, de s'être bien entraîné, de s'être bien arraché les tripes en fait, et puis c'est surtout là après quand on sort, on ne sent plus rien: le froid, le chaud, une douleur, non, ça n'existe plus quoi. Et ça, c'est un petit peu... c'est même très proche de la drogue, faut dire ce qui est, c'est quand même très bon, c'est pour ça qu'il y a beaucoup de gens qui s'y laissent prendre. C'est ensuite l'apaisement immédiat que procure le produit dès les premiers instants qui est décrit.

La plupart n'utilisent pas la voie intraveineuse. Ils sniffent ou fument l'héroïne. Les premières consommations ne sont pas toujours suivies d'un engagement immédiat dans la consommation. Parfois même, il s'écoule quelques années avant que les personnes ne reprennent de l'héroïne. Mais c'est alors le début d'un parcours de consommation semblable à celui de la plupart des héroïnomanes (Leroux, 2002).

En fait, bien sûr, à partir d'aussi peu de cas, il est impossible de lier le sport de performance et les toxicomanies. Mais l'on peut remarquer que toutes les personnes concernées se sont investies dans le sport comme s'il était le seul moyen de se dégager une identité gratifiante. Leur engagement dans l'héroïnomanie ne se produit que lorsque le sport ne leur procure plus les satisfactions nécessaires ou lorsqu'il rentre en conflit avec d'autres domaines de la vie en société.

\section{Essai de typologie des usages}

À travers ce bref passage en revue des différents usages de produits psychoactifs rencontrés dans les activités sportives institutionnelles, on peut distinguer des logiques fort différentes.

- Les produits psychoactifs peuvent fonctionner comme désinhibiteurs sans que le sport perde sa propre fonction de libération des émotions. Dans ce cas, souvent, la valeur accordée à l'exercice sportif est plus proche du ludique que de la performance et même si les consommations sont régulières, voire quotidiennes, elles restent modérées et correspondent à des temporalités précises de la vie quotidienne.

- D'autres usages prennent la forme d'excès de produits psychoactifs, parfois ponctuels, parfois prolongés, qui suivent les temporalités de l'exercice. Ces excès sont considérés comme normaux et sont relativement admis dans le milieu sportif, quelle que soit leur intensité. Ces usages sont aussi clairement liés à la décompression. Mais ici, comme l'exercice sportif est plus intense et peu propice à la libération des émotions, les produits psychoactifs apparaissent comme un des médiateurs possibles de cette libération. On sait que les jeunes qui ont des pratiques sportives intensives consomment davantage de produits psychoactifs que ceux qui ont une pratique modérée, c'est-à-dire celle qui est la plus susceptible de correspondre à la fonction de décompression ${ }^{3}$ (Choquet et al., 1999).

La liaison apparaît pour l'alcool et les produits illicites, mais non pour le tabac. Une même liaison s'opère au niveau des comportements violents. 
Certains usages encore ont clairement une valeur de dopage: il s'agit de faire mieux que ce que l'on devrait faire quelle que soit la situation: manque de préparation, blessure, stagnation des résultats, volonté de passer à un niveau supérieur.

Enfin, on trouve des usages toxicomaniaques qui prennent place lorsque la gratification disparaît, pendant ou à la fin de la carrière sportive.

Comment arriver à donner sens et forme à ces usages? On voit bien qu'ils correspondent à différentes positions dans la gamme des activités sportives et qu'entre la libération des émotions et l'intensité des pratiques se joue un arbitrage subtil qui fait varier les usages. Si l'on veut comprendre quelles sont les logiques de ces usages, il est nécessaire de les classer en fonction des effets recherchés par l'usager lui-même. Les définitions ou les catégorisations que donnent certains chercheurs peuvent alors nous servir de soubassement, même s'ils ne se réfèrent pas à des activités sportives. Ainsi, Alain Ehrenberg a proposé de retenir trois lignes de pratique qui ne recoupent pas entièrement la distinction drogues dures/drogues douces, car elles peuvent s'investir dans n'importe quel produit: celle du trou noir, de la sociabilité et du dopage (un joint avant l'école, un fix avant le travail) (Ehrenberg, 1995, 132). Pourtant, cette partition n'est pas satisfaisante, la différence entre dopage et sociabilités n'apparaissant pas clairement. En effet, si certains produits sont consommés dans des situations récréatives, c'est bien que ces produits permettent d'avoir des relations sociales plus agréables en permettant le relâchement des autocontrôles. En tant que telle, leur prise pourrait s'apparenter à une forme de dopage, puisqu'elle facilite les relations sociales par ses effets relaxants et désinhibiteurs.

Les distinctions souvent faites entre les usages amicaux et conviviaux, les usages de dopage et les usages compulsifs (le trou noir) des produits psychoactifs désignent finalement des valeurs sociales. Les usages festifs ou récréatifs sont acceptables puisque les personnes restent dans le lien social et ne font finalement que s'accorder une 'pause', ce qui est à l'opposé des usages chroniques où les personnes sortent du lien social en établissant une relation quasi exclusive avec leur(s) produit(s).

Dans le cas du dopage, les usages s'apparentent à de la «triche»; ils visent à permettre de meilleures performances à l'aide d'un adjuvant chimique. Les étudiants bousculés par des périodes d'examen prolongées dont dépend leur avenir peuvent ainsi recourir à différents produits d'une manière qui peut évoquer le dopage ${ }^{4}$. De même, dans les métiers de la création, il arrive que soient consommés des produits en vue de trouver l'inspiration. Pourquoi ces situations ne sont-elles pas considérées comme de la fraude alors que c'est le cas dans les compétitions sportives? C'est bien que le sport représente un idéal égalitaire (Ehrenberg, 1991) qui n'a pas cours dans la société de marché. Les compétiteurs sont considérés comme égaux et la responsabilité de leurs actes leur incombe.

La recherche du plaisir, inhérente à tout être humain, se situe donc dans des contextes différents, plus ou moins admis socialement. De la simple détente en rupture avec les tensions de la vie quotidienne à la décompression plus importante recherchée après des périodes d'effort intense, du plaisir que procure la réalisation de performances, assistées ou non par des produits, à l'abandon dans une relation quasi exclusive à un produit, c'est bien cette quête qui se décline ici dans toutes ses formes.

Deux modalités d'utilisation émergent des situations que nous avons examinées. Toutes deux prennent forme par rapport aux activités de la vie quotidienne (qu'il s'agisse

Lors de l'émission «Zinzin», du lundi 14 juin 2000, 20 h 10 - 21 h 30, sur France Inter, consacrée aux problèmes du dopage en milieu sportif, un jeune auditeur appelle pour expliquer qu'il a fumé un joint la veille du bac pour se détendre. J'ai bien déliré et j'ai eu seize conclut-il. 
ou non d'activités sportives), mais y répondent de manière différente. L'une accompagne ces activités en fournissant des ressources qui les rendent moins contraignantes, l'autre sert à construire ou à renforcer des temps de rupture par rapport à ces activités.

Dans le premier cas, on peut parler d'usage de routine, non pas que les usages dont il est question ici soient quotidiens ou même réguliers, mais bien qu'ils répondent aux activités routinières de la vie. Ils soulagent de manière modérée des tensions résultant du contrôle et de la gestion des affects retenus dans l'exercice des charges quotidiennes. On trouve là des usages individuels aussi bien que collectifs.

Dans le second cas, on peut parler d'usage de rupture. Les utilisations des produits s'articulent alors à un contexte précis, spatial et temporel, qui est organisé pour faciliter le relâchement des émotions et qui brise la continuité des activités routinières. On retrouvera là les fêtes de fin d'année, les excès de vacances, les troisièmes mi-temps, etc. L'excès est une des caractéristiques des usages de rupture, il est le reflet inversé de la retenue et de la modération des usages de la vie courante. Ces usages sont essentiellement collectifs et prennent corps dans des sociabilités préexistantes.

Ces deux modalités d'utilisation des produits psychoactifs compensent les tensions vécues au jour le jour et ne sont compréhensibles que par rapport aux situations dans lesquelles les personnes se trouvent et qui les affectent plus ou moins.

Pourtant, il existe aussi des utilisations qui sont à la fois excessives et fréquentes. Elles peuvent correspondre à un comportement individuel (toxicomanie) ou à une normalisation de l'excès dans un groupe, mais il est improbable qu'elles n'aient aucun rapport avec les activités sociales de l'individu ou du groupe. On peut qualifier cette utilisation de «routine de l'excès ».

Ces différentes utilisations ne rendent cependant pas compte réellement des relations entre les situations dans lesquelles se trouvent les personnes et leurs consommations. Si, nécessairement, chaque personne peut avoir un comportement fort différent en fonction de son parcours, il semble que l'on peut aussi retrouver là des logiques sociales.

Les usages ne peuvent être pensés en dehors des contextes sociaux dans lesquels ils prennent forme. Pourtant, il ne s'agit pas tant de considérer des conditions sociales en tant que telles, mais plutôt de voir comment celles-ci créent des tensions qui agissent sur les personnes et rendent nécessaire l'utilisation d'un adjuvant chimique de l'action (Ehrenberg, 1995, 127-128). Ces tensions s'exercent forcément entre une action et son résultat, dans le rapport entre l'effort accompli et la satisfaction qui en découle. Il est donc bien question ici de positions sociales et de conditions de vie, des situations occupées et des tensions qui en résultent.

J'ai donc essayé de bâtir une typologie qui puisse rendre compte des différents usages par rapport aux situations dans lesquels ils naissent. La réflexion dépasse alors les consommations des seuls sportifs et je me suis servi aussi d'exemples tirés d'autres recherches pour approfondir ce cadre d'analyse. La typologie que je propose comporte quatre catégories, définies par la relation avec le(s) produit(s) psychoactif(s) utilisé(s) et les effets recherchés en relation avec les situations occupées et les tensions qui leur sont inhérentes: il s'agit du confort, de la résistance, de la performance et de l'addiction. Les deux premières catégories regroupent les usages liés à la décompression selon qu'ils varient en intensité.

Dans le confort, on trouvera aussi bien les usages récréatifs du cadre qui aime à boire un whisky ou deux le soir après le travail que ceux du jeune qui fume un ou deux joints après l'école. Il en est de même pour le sportif qui cherche à se relaxer après une longue journée d'entraînement. On y trouvera aussi des usages encore davantage liés au travail, tels que ceux de certains fumeurs de cannabis qui grillent un joint soigneusement dosé 
avant d'arriver au travail (Aquatias et al., 1997). Il s'agit ici de se détendre et de se soulager de la tension des activités professionnelles, une tension qui n'est pas très importante mais qui n'en nécessite pas moins un minimum d'aide pour se relâcher. Cette aide pourrait aussi bien se retrouver dans d'autres pratiques: voir un bon film ou aller jouer au football avec les copains en bas des immeubles sont des activités qui correspondent aussi à ce nécessaire relâchement de la gestion des émotions. Ces usages sont essentiellement des usages de routine, associés aux activités de la vie quotidienne et ne concernent pas des consommations intensives.

La résistance concerne un niveau plus élevé de tension: c'est quand la pression pèse plus lourdement sur les personnes que les usages deviennent plus conséquents, voire excessifs. On y trouvera plusieurs types de situations, selon qu'il s'agit d'utilisation de routine ou de rupture.

Les utilisations de routine concernent des consommations à la fois importantes et quotidiennes. L'ennui qui pèse sur les adolescents et les jeunes adultes des cités défavorisées de banlieue (Aquatias et al., 1997) ou sur les travailleurs exécutants dans des professions peu qualifiées ou peu intéressantes et où seules règnent la déresponsabilisation et la monotonie en constitue un versant. L'autre est celui des personnes qui doivent arriver à maintenir un niveau de production ou d'attention pour garder leur position: certains métiers à forte responsabilité comme ceux de la sécurité et de la sûreté (Haguenoer, Hannothiaux, Lahaye-Roussel, Fontaine, Legrand, Shirali et al., 1997), certaines professions où les heures supplémentaires sont foison, certains postes que les personnes ne peuvent espérer garder que si leur rentabilité ne diminue pas ${ }^{5}$. On trouvera là aussi les usages de dopants des sportifs blessés qui veulent conserver leur place.

Les usages de rupture se retrouvent dans des situations où l'écart entre l'effort des activités quotidiennes et la satisfaction que l'on y trouve est conséquent, que l'effort soit très important, la gratification faible ou que les deux éléments se conjuguent.

C'est dans ces situations que l'on trouvera des usages festifs d'autant plus intensifs que la vie quotidienne n'offre que peu de gratification. Ainsi Sandy Queudrus parle à propos des jeunes fréquentant les free-parties de «gestion du déclassement» pour des personnes qui sont globalement issues des milieux populaires, disposent d'un niveau d'études modeste et se trouvent dans des situations sociales incertaines et précaires (Queudrus, 1998). C'est là aussi que les usages festifs démesurés des sportifs, qui compensent la constance des efforts pendant la journée, la compétition ou la saison, prendront tout leur sens. Et par ailleurs, il semble que leur situation, dans le domaine du sport, soit tout aussi incertaine et précaire par son incessante remise en jeu, même si la gratification est certes plus importante ici.

Dans les usages de routine comme dans les usages de rupture, il ne s'agit pas d'augmenter des performances, même si les usages sont des conduites dopantes. Il s'agit ici de supporter les difficultés et non de les dépasser. La résistance est un mode de compensation.

La performance est tout autre. Il ne s'agit pas ici de supporter mais bien d'aller plus loin, de se dépasser et de faire mieux. Si la résistance concerne ceux qui veulent arriver à tenir dans la situation qui est la leur, soit qu'elle les satisfasse, soit qu'ils n'en imaginent pas d'autres, la performance vise à dépasser la position présente. On trouvera ici non seulement

Patrick Laure cite une recherche accomplie par C. Buisset, A. Hiault, L. Mignien, S. Volkoff, C. Monfort, «consommation de médicaments psychoactifs chez les salariés occupant des emplois administratifs», $\hat{A} g e$, travail, santé, Paris, Inserm, 1996, dans laquelle les auteurs trouvent un lien entre les consommations de médicaments «pour les nerfs» et les composantes de pression et de sollicitation sur les lieux de travail (Laure, 2000, 193). 
les prises de stéroïdes des adolescents qui désirent se muscler et les consommations de produits dopants des sportifs ou des équipes qui veulent obtenir un classement supérieur, mais aussi les utilisations de cocaïne ou d'amphétamines dans les affaires et le show-business, les absorptions d'hallucinogènes des créateurs en quête d'inspiration ou des mystiques en quête de révélation. Les usages sont essentiellement des usages de routine. La rupture semble peu fréquente ici.

L'addiction correspond aux définitions classiques de la toxicomanie. Elle se spécifie par la dépendance et peut faire suite aux types précédents d'usage. On y retrouvera aussi bien des dépendances aux produits psychoactifs et dopants, à l'alimentation, aux jeux, etc. L'usage de rupture est routinier, la routine est de bâtir de la rupture. Comme dans la résistance, on a affaire à une routine de l'excès, mais ici celle-ci ne s'intègre pas à d'autres activités. Il y a un changement de polarité: la consommation devient l'activité principale, autour de laquelle gravitent toutes les autres activités.

Pour résumer, et de manière un peu caricaturale, on peut dire que les usages de confort correspondent au relâchement de soi, les usages de résistance au maintien de soi, les usages de performance au dépassement de soi, les usages d'addiction à l'oubli de soi.

Cette typologie est cependant loin d'être parfaite. Certains cas précis restent difficiles à classer: qu'en est-il par exemple des situations où, pour tenir leur place, les individus sont obligés d'améliorer sans cesse leurs résultats et, en conséquence, consomment des produits psychoactifs ? Est-ce de la performance ou de la résistance? Qu'il s'agisse de certains sports où l'évolution des modes d'entraînement produit une augmentation rapide des performances ou de certains secteurs en pointe de la vente où il est nécessaire d'accroître sans cesse le chiffre d'affaires pour tenir sa place, il est relativement difficile de spécifier dans quelle catégorie on classerait ces usages. Mais indubitablement cette zone de trouble évoque aussi des effets de champ particuliers où existe une relative confusion entre le maintien de soi et le dépassement de soi. C'est notamment le cas des activités sportives.

De même, certains usages peuvent se cumuler. Rien n'empêche un sportif de bon niveau de se livrer à quelques excès festifs et de consommer dans le même temps des produits à fin de performance, comme le font certains «teufeurs» qui, en rave party, consomment des produits démultipliant les sensations dans le même temps qu'ils consomment des amphétamines pour profiter plus longtemps de la fête (Queudrus, 1998; Racine, 1999).

Enfin, il reste à définir avec davantage de clarté les critères (tensions résultant des situations sociales et/ou personnelles, intensité de consommation, etc.) qui séparent confort et résistance, résistance et dopage, etc.

Ces quatre catégories sont donc encore bien incertaines et demandent des compléments évidents. Si je les présente néanmoins ici, c'est parce qu'elles permettent de souligner plusieurs aspects essentiels des consommations de produits psychoactifs.

- S'il existe des trajectoires personnelles qui peuvent prédisposer aux usages, les situations sociales jouent aussi un rôle. Ces situations sociales ne doivent pas être comprises en terme d'état (être ouvrier, champion ou jeune), mais en terme de tension entre une situation vécue et un ensemble de contraintes objectives. Or, la prévention des usages de produits psychoactifs est toujours davantage dirigée vers des individus ou des catégories que vers des situations, comme si, justement, les situations étaient égales. On trouve par exemple la volonté dans la prévention de l'alcoolisme dans l'entreprise de ne pas désigner de population cible pour respecter la dignité des personnes (Huez, 1990), sans qu'il soit reconnu que certaines conditions de travail peuvent contribuer au développement de certaines consommations. 
- Parce qu'il s'agit de catégories descriptives, elles permettent de mieux comprendre à quels enjeux les personnes sont confrontées et ensuite de faire sortir les pratiques des cadres juridiques et médicaux, nécessairement normalisateurs, dans lesquelles elles sont analysées habituellement. Le sport est, de plus, souvent soumis à une idéologie égalitaire, à la réalité pourtant assez improbable, qui ne peut rendre compte des différentes tensions qui jouent sur les personnes bien au delà de leurs capacités physiques.

- Enfin, cette description s'inscrit dans une configuration précise d'usages, liée aux transformations de la société. Les quatre catégories proposées prennent corps dans la tension entre souffrance et performance. Et si des cas limites, que j'ai signalés, se détachent parfois, c'est que dans certaines conjonctions, des personnes souffrent de ne pas être performantes, dans une société où la performance est une forte valeur sociale (Ehrenberg, 1991). Dans ce contexte, la typologie que je propose permet alors de mieux saisir les effets de système qui pèsent sur les gens.

\section{Conclusion: des consommations de produits psychoactifs aux fonctions du sport}

Si l'on reprend notre question de départ sur les liens entre activités sportives et consommation de produits psychoactifs, on peut voir se détacher assez nettement certaines relations:

- Les usages de produits psychoactifs peuvent venir à l'appui des activités sportives aussi bien quand celles-ci sont d'ordre ludique que lorsqu'elles tiennent de la carrière. Ils ne font que reproduire des usages qui ont lieu dans d'autres domaines d'activités et ne peuvent être examinés dans l'unique domaine sportif comme si celui-ci était totalement disjoint du reste de la société et des transformations qui l'agitent.

- Produits psychoactifs et activités sportives peuvent être utilisés conjointement pour obtenir des effets de libération du contrôle des émotions. Leurs utilisations respectives sont susceptibles de varier en fonction du rapprochement entre activité sportive et carrière sportive.

- Le dopage dans le sport peut correspondre à des fins très différentes et il semble nécessaire de mieux comprendre les situations particulières dans lesquelles des conduites dopantes naissent.

Ces relations ne peuvent cependant être expliquées qu'à travers une vision plus large qui évoque à la fois des effets de champ propres au monde sportif et à la fois les modifications que les changements de la société répercutent dans la société sportive.

Les effets de champ tiennent essentiellement à la normalisation de certaines consommations d'excès par l'idéologie sportive. Cette normalisation suit les logiques sociales d'excès qui sont souvent à l'œuvre dans les situations festives. Elle se déploie cependant en suivant des cultures de consommation qui peuvent être propres au sport. Ainsi l'excès apparaît dans certains discours comme une valeur supplémentaire, une «plus-value» que l'on apporte à l'effort sportif. Nous avons vu comment certaines consommations pendant l'entraînement ou les compétitions étaient liées au handicap supplémentaire ainsi constitué. Mais la pratique sportive elle-même servira d'alibi à l'excès comme les propos de ce sportif le laissent bien entendre: 
Mais je supportais bien, même quand j'étais au CREPS de X. On faisait la fête tous les soirs, on buvait comme des gogols, on buvait, on fumait, avec l'équipe de France espoir. Tous les soirs on était déchiré, et la journée, le lendemain midi, on était sur le terrain pour nous entraîner. C'est pour ça, il y a des gens, ils hallucinent quoi. Je prenais des cuites des fois, moins maintenant, mais je prenais des cuites le soir. Le lendemain je pouvais aller bosser. Les gens prenaient deux jours à se remettre. L'avantage avec le sport c'est que ton corps est plus résistant, ou il récupère vite.

C'est parce que l'on est sportif et que le corps est donc «sain » que l'on peut s'accorder des dépassements plus importants.

L'admissibilité de ces conduites dans le milieu sportif est cautionnée par l'effort accompli et si l'on ne consomme pas forcément ouvertement, il est clair que l'encadrement ne cherche pas réellement à contrôler ces consommations. On trouve là des valeurs qui recoupent celles de l'activité sportive: ne pas s'écouter, repousser les limites, etc. Mais ces effets de champ sont aussi concomitants au déploiement de certaines dimensions du modèle libéral dans notre société. La performance devient une métaphore de la productivité et le sport de compétition un modèle de l'entreprise.

Parallèlement, le rétrécissement du marché du travail a joué de manière simultanée dans plusieurs dimensions. En réduisant les perspectives des jeunes, il a accru la tension qui pèse sur eux à travers la réussite scolaire, considérée comme un préalable à toute insertion professionnelle. L'activité scolaire était déjà un véritable travail, mais les enjeux qui s'y attachent ont sensiblement augmenté. Un certain nombre d'usages du cannabis notamment sont directement liés à la montée en puissance de cette pression de l'institution scolaire, relayée par la famille et les médias. L'augmentation des tensions de la vie scolaire suppose des espaces de décompression en conséquence. Les jeunes mettent ainsi souvent en balance les joies de la pratique sportive face aux contraintes scolaires. Au demeurant, la popularité d'un certain nombre de champions accentue l'attirance du sport et, dans les milieux populaires défavorisés, la réduction des perspectives professionnelles et la médiatisation du succès de certains champions crée un appel incessant vers la pratique sportive de compétition. Le déploiement des pratiques sportives juvéniles, en club ou hors club, correspond au moins autant à la nécessité de créer des espaces de décompression qu'à l'envie de trouver un espace gratifiant, voire à l'espoir d'être un jour champion. En conséquence, les motivations mêmes des jeunes sont relativement ambiguës et la différence entre la fonction de décompression et la fonction professionnalisante des activités sportives est assez trouble.

Dans le même temps, la diminution des capacités du marché du travail a provoqué une professionnalisation de sphères d'activité peu ou pas investies. Le champ du sport, notamment, a ainsi subi une professionnalisation importante alors qu'il était auparavant essentiellement tenu par des bénévoles ${ }^{6}$. De plus, les problèmes de violence dans les banlieues ont créé un véritable marché de l'emploi subventionné par les municipalités et l'État. Ce mouvement de professionnalisation s'est prolongé par la création, ces dernières années, de filières spécialisées vers les métiers du sport. En conséquence, beaucoup de personnes ayant eu une carrière sportive interrompue précocement travaillent dans l'encadrement sportif, transmettant ainsi les valeurs de compétition qu'ils ont eux-mêmes intériorisées.

6 Les récents changements législatifs réduisent les marges de manœuvre dont disposaient les clubs pour rétribuer certains joueurs par leur participation à l'encadrement et incitent d'autant plus à la professionnalisation. 
La conjonction de ces phénomènes contribue à reproduire l'idéologie sportive liée à la compétition dans d'autres types d'activités sportives et notamment dans les activités de sport liées à l'éducation, qu'elles se passent en club ou soient dirigées vers les jeunes des milieux défavorisés au travers de protocoles spécifiques. Le niveau éducatif et le niveau de production des champions, notamment, se confondent souvent dans l'esprit des entraîneurs comme dans l'esprit des jeunes eux-mêmes. L'apprentissage des enfants au sport est souvent le même, qu'il s'agisse de sport à visée éducative ou de sport à visée de performance ${ }^{7}$. On s'interroge bien peu sur les méthodes de transmission du contrôle de soi et cette dimension, dans certaines activités éducatives de vacances, se limite souvent à contrôler le langage des enfants et à éviter les disputes. Dans les clubs, l'apprentissage des exercices se lie rarement aux apports de ceux-ci dans la vie quotidienne, rendant caduc le caractère mimétique des activités sportives dans le domaine de l'éducation.

C'est ce qui explique que le modèle tracé par Norbert Elias nous apparaisse relativement inopérant à présent. Ses limites se trouvent au niveau de la confusion entre les différentes fonctions du sport: non pas seulement entre joueurs et spectateurs, mais de manière bien plus complexe entre la fonction éducative et la fonction professionnalisante. Les usages de produits psychoactifs en fonction des types d'investissement dans le sport montrent que si les activités sportives ne suffisent plus toujours à compenser la répression des émotions, c'est bien qu'alors même qu'il n'est pas pratiqué à un haut niveau, il est investi de manière conséquente par les jeunes et correspond moins à une libération des tensions de la vie quotidienne. Le recours aux produits psychoactifs peut alors intervenir, à côté des activités sportives ou à l'intérieur même de celles-ci. Il se produit un décalage entre l'idéologie sportive et les pratiques sportives, décalage qui naît de l'augmentation des tensions auxquelles sont soumises les personnes dans la société tout autant que de la diminution de l'efficacité du jeu sportif comme espace de libération des émotions.

\author{
Sylvain Aquatias \\ aqtias@ club-internet.fr \\ 14, rue Eugène Sue \\ F-75018 Paris
}

\title{
BIBLIOGRAPHIE
}

AQUATIAS S., 1998-1999, Analyse de 272 questionnaires passés en milieu techno, démarche de "prévention par les pairs», Mission Départementale de Prévention des Toxicomanies, Conseil général de Seine-Saint-Denis.

AQUATIAS S. (dir.), BOITEL L., GRENOUILLET G., 2002, Les consommations de produits psychoactifs dans les milieux festifs de la culture rock, Paris, RES, OFDT.

AQUATIAS S., KHÉDIM H., MURARD N., GUENFOUD K., 1997, L'usage dur des drogues douces, recherche sur la consommation de cannabis dans la banlieue parisienne, Paris, GRASS-CNRS.

AQUATIAS S. (dir.), LEROUX M., DESRUES I., VALLETTE VIALLARD C., STETTINGER V., 1999, Activités sportives, pratiques à risques, usages de substances dopantes et psychoactives: recherche sur la pratique moderne du sport, Paris, RESSCOM.

BROMBERGER C., HAYOT A., MARIOTTI J.-M., 1987, Allez l'O-M, Forza Juve - La passion pour le football à Marseille et à Turin, Terrain, 8, 8-41.

7 À l'exception notable d'un certain nombre d'associations qui pratiquent l'insertion par le sport et dont les conceptions pédagogiques sont fort différentes (Aquatias, 1999). 
CHOQUET M. et al., 1999, Jeunes, sport, conduites à risques, Paris, ministère de la Jeunesse et des sports.

EHRENBERG A., 1991, Le culte de la performance, Paris, Calmann-Lévy.

EHRENBERG A., 1995, L'individu incertain, Paris, Calmann-Lévy.

ELIAS N., 1973, La civilisation des mœeurs, Paris, Calmann-Lévy.

ELIAS N., 1975, La dynamique de l'Occident, Paris, Calmann-Lévy.

ELIAS N., 1996, Du temps, Paris, Fayard.

ELIAS N., DUNNING E., 1994, Sport et civilisation, la violence maîtrisée, Paris, Fayard.

GOFFMAN E., 1974, Les rites d'interaction, Paris, Minuit.

HAGUENOER J.M., HANNOTHIAUX M.H., LAHAYE-ROUSSEL M.C., FONTAINE B., LEGRAND P.M., SHIRALI P. et al., 1997, Prévalence des comportements toxicophiles en milieu professionnel: une étude dans la région Nord-Pas-de-Calais, Bulletin de l'Ordre des médecins, 80, 11-5.

HUEZ D., 1990, CHSCT et médecine du travail: deux éléments indispensables pour la prévention de l'alcoolisme en milieu de travail, in CARO G. (Ed.), De l'alcoolisme au bien boire, tome 2, Paris, l'Harmattan, 108-115.

HUIZINGA J., 1951, Homo Ludens; essai sur la fonction sociale du jeu, Paris, Gallimard.

LAURE P., 2000, Dopage et société, Paris, Ellipses.

LEROUX M., Consommations intégrées et sport de haut niveau, in FAUGERON C., KOKOREFF M. (dir.), Société avec drogue, Toulouse, Erès, 2002, 79-97.

QUEUDRUS S., 1998, La free-party, une gestion du déclassement, Mémoire de DEA en sociologie, Paris, Université de Paris VIII.

RACINE É., 1999, Pratiques culturelles et prise de risques chez les jeunes en milieu techno, ministère de la Jeunesse et des Sports, CRIPS, Île-de-France. 